\title{
Combined numerical-experimental model for the identification of mechanical properties of laminated structures
}

\author{
A.L. Araújo ${ }^{\text {a }}$, C.M. Mota Soares ${ }^{\text {b,* }}$, M.J. Moreira de Freitas ${ }^{\text {c }}$, P. Pedersen ${ }^{\text {d, }}$, \\ J. Herskovits ${ }^{\text {e }}$ \\ ${ }^{a}$ ESTIG, Campus de Sta., Instituto Politécnico de Bragança, Apolónia, Apartado 134, 5301-857 Bragança, Portugal \\ ${ }^{\mathrm{b}}$ IDMEC, Instituto de Engenharia Mecânica, I.S.T., Av. Rovisco Pais, 1049-001 Lisboa, Portugal \\ ${ }^{c}$ ICEMS-UME, Dept. Eng. Mecânica, I.S.T., Av. Rovisco Pais, 1049-001 Lisboa, Portugal \\ ${ }^{\mathrm{d}}$ Department of Solid Mechanics, Building 404, Technical University of Denmark, DK-2800 Lyngby, Denmark \\ e COPPE, Universidade Federal do Rio de Janeiro, Caixa Postal 68503, 21945-970 Rio de Janeiro, Brazil
}

\begin{abstract}
A combined numerical-experimental method for the identification of six elastic material modulus of generally thick composite plates is proposed in this paper. This technique can be used in composite plates made of different materials and with general stacking sequences. It makes use of experimental plate response data, corresponding numerical predictions and optimisation techniques. The plate response is a set of natural frequencies of flexural vibration. The numerical model is based on the finite element method using a higher-order displacement field. The model is applied to the identification of the elastic modulus of the plate specimen through optimisation techniques, using analytical sensitivities. The validity, efficiency and potentiality of the proposed technique is discussed through test cases. (C) 2000 Elsevier Science Ltd. All rights reserved.
\end{abstract}

Keywords: Identification; Mechanical properties; Numerical-experimental vibrations; Sensitivity analysis; Optimisation

\section{Introduction}

The dynamic free vibration behaviour of a structure made of anisotropic materials depends on its geometry, density, boundary conditions and elastic constants. Hence, a non-destructive method for the determination of the elastic constants of the materials that make up the structure can be developed. The method combines experimental results of a free vibration test with a numerical model capable of predicting the dynamic free vibration behaviour of the structure and makes use of optimisation techniques.

Thus, it becomes necessary to use an error measure that expresses the difference between experimental and numerical eigenvalues. This error measure is then minimised with respect to the elastic modulus of the different materials.

This work is a generalisation of the work presented by Mota Soares and coworkers (see e.g., $[1,2])$ using the

\footnotetext{
${ }^{*}$ Corresponding author. Tel.: +351-21841-7455; fax: +351-218417915.

E-mail address: cmmsoares@alfa.ist.utl.pt (C.M. Mota Soares).
}

Mindlin plate theory along with a finite element model, and Araújo et al. [3] using a higher-order displacement finite element model. It has the innovative aspect of contemplating identification of mechanical parameters of specimens having layers made up of several materials and general stacking sequences. It is closely related to the developments presented by Pedersen [4] for thin plates using the classical plate theory and Frederiksen [5] for thick plates using a higher-order theory associated to a numerical model based on the Rayleigh-Ritz approach for symmetric lay-ups and specimens made of a single material. Other research works that also use the Rayleigh-Ritz method along with the classical plate theory were presented by Wilde and Sol [6], Sol [7] and Wilde [8], using Bayesian estimation instead of optimisation techniques for symmetric single material laminates. Lai and Ip [9] presented a method, using the Kirchhoff plate theory, which takes into account both the confidence associated with mathematical modelling and parameter estimates. This last technique has been validated satisfactorily on symmetric single material laminates. In fact, in more recent works, priority is given to assessment of uncertainties. In [10], these questions are addressed based on statistics. 
More recently, a method based on response surfaces has been used by Rikards and coworkers (see e.g., $[11,12])$ and Bledzki et al. [13]. This technique commonly called planning of experiments was used successfully to identify elastic properties in unidirectional laminates.

The use of model updating techniques for the identification of mechanical properties of laminates was also presented by Cunha and Piranda [14], applied to sandwich composite structures.

An overview of different approaches on combined numerical-experimental identification methods with the goal of obtaining material stiffness for composite structures, based on eigenfrequencies and optimisation, is carried out by Pedersen [15].

\section{Numerical model}

The eigenvalue problem and the sensitivity analysis are carried out using a third-order shear deformation theory whose pioneering works are described in [16,17] and has been applied to discrete finite element models by Mallikarjuna and Kant [18], among others. Full details regarding the model development and implementation for dynamics can be found in $[19,20]$.

Fig. 1 shows a rectangular plate of constant thickness $h$ and plane dimensions $a$ and $b$. A Cartesian coordinate system $(x, y, z)$ is located at the middle plane as shown.

The assumed displacement field is a third-order expansion in the thickness coordinate for the in-plane displacements and a constant transverse displacement:

$$
\begin{aligned}
u(x, y, z, t)= & u_{0}(x, y, t)+z \theta_{x}(x, y, t) \\
& +z^{2} u_{0}^{*}(x, y, t)+z^{3} \theta_{x}^{*}(x, y, t), \\
v(x, y, z, t)= & v_{0}(x, y, t)+z \theta_{y}(x, y, t) \\
& +z^{2} v_{0}^{*}(x, y, t)+z^{3} \theta_{y}^{*}(x, y, t), \\
w(x, y, z, t)= & w_{0}(x, y, t),
\end{aligned}
$$

where $u_{0}, v_{0}$ and $w_{0}$ are the in-plane displacements in the $x$-, $y$ - and $z$-directions and $\theta_{x}$ and $\theta_{y}$ are the rotations of

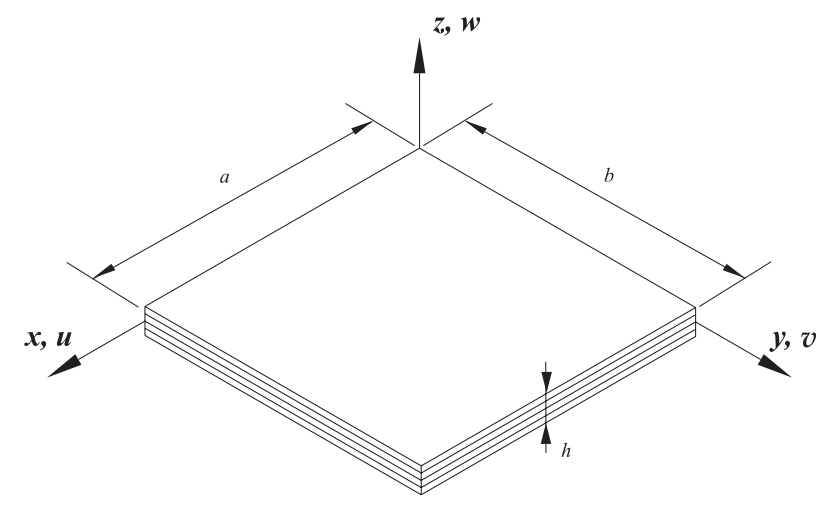

Fig. 1. Plate global coordinate system $(x, y, z)$ and associated displacement field $(u, v, w)$. normals to the midplane about the $y$-axis (anticlockwise) and $x$-axis (clockwise), respectively. The functions $u_{0}^{*}, v_{0}^{*}, \theta_{x}^{*}$ and $\theta_{y}^{*}$ are higher-order terms in the Taylor series expansion, defined also in the midplane of the plate.

As for the constitutive relations, considering a plane stress analysis and a linear elastic material, it can be shown that, for a laminae of orthotropic material in the $(1,2,3)$ coordinate system (Fig. 2$)$, the stress-strain relation is

$\boldsymbol{\sigma}_{(1,2,3)}=\mathbf{Q} \boldsymbol{\varepsilon}_{(1,2,3)}$

with the following constitutive matrix:

$\mathbf{Q}=\frac{E_{1}}{8 \alpha_{0}}\left[\begin{array}{ccccc}8 & \alpha_{4}-\alpha_{3} & 0 & 0 & 0 \\ \alpha_{4}-\alpha_{3} & 8-2 \alpha_{2} & 0 & 0 & 0 \\ 0 & 0 & \alpha_{8}-\alpha_{9} & 0 & 0 \\ 0 & 0 & 0 & \alpha_{8}+\alpha_{9} & 0 \\ 0 & 0 & 0 & 0 & \frac{1}{2}\left(8-\alpha_{2}-3 \alpha_{3}-\alpha_{4}\right)\end{array}\right]$

The non-dimensional parameters in Eq. (3) are defined as $[5,21]$

$$
\begin{aligned}
& \alpha_{2}=4-4 E_{2} / E_{1}, \\
& \alpha_{3}=1+\left(1-2 v_{12}\right) E_{2} / E_{1}-4 \alpha_{0} G_{12} / E_{1}, \\
& \alpha_{4}=1+\left(1+6 v_{12}\right) E_{2} / E_{1}-4 \alpha_{0} G_{12} / E_{1}, \\
& \alpha_{8}=4\left(G_{13}+G_{23}\right) \alpha_{0} / E_{1}, \\
& \alpha_{9}=4\left(G_{13}-G_{23}\right) \alpha_{0} / E_{1},
\end{aligned}
$$

where $\alpha_{0}=1-v_{12}^{2} E_{2} / E_{1}$ and $E_{1}, E_{2}$ are Young's modulus in (1,2)-direction (Fig. 2), $G_{12}, G_{23}$ and $G_{13}$ are the transverse shear modulus in planes $1-2,2-3$ and $1-3$ (3 perpendicular to $1-2$ plane), respectively, and $v_{12}$ is the major Poisson's ratio.

The inverse relations of Eq. (4) can be written as

$$
\begin{aligned}
& E_{2} / E_{1}=\left(4-\alpha_{2}\right) / 4, \\
& G_{12} / E_{1}=\left(8-\alpha_{2}-3 \alpha_{3}-\alpha_{4}\right) /\left(16 \alpha_{0}\right), \\
& v_{12}=\left(\alpha_{4}-\alpha_{3}\right) /\left(8-2 \alpha_{2}\right), \\
& G_{13} / E_{1}=\left(\alpha_{8}+\alpha_{9}\right) /\left(8 \alpha_{0}\right), \\
& G_{23} / E_{1}=\left(\alpha_{8}-\alpha_{9}\right) /\left(8 \alpha_{0}\right), \\
& \alpha_{0}=1-\left(\left(\alpha_{4}-\alpha_{3}\right)^{2} /\left(16\left(4-\alpha_{2}\right)\right)\right) .
\end{aligned}
$$

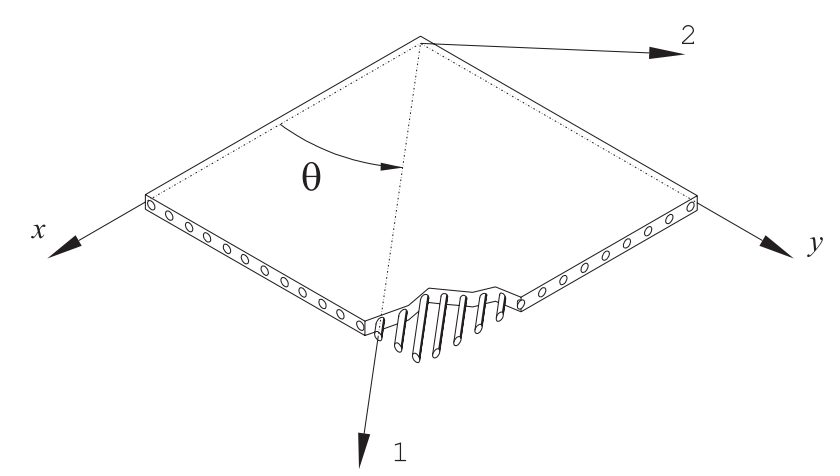

Fig. 2. Orthotropic material laminae; principal $(1,2)$ and global $(x, y)$ coordinate system. 
For a laminae in the $(x, y, z)$ coordinate system (Fig. 2), the stress-strain relations are

$\boldsymbol{\sigma}_{(x, y, z)}=\frac{E_{1}}{8 \alpha_{0}} \mathbf{c} \cdot \boldsymbol{\varepsilon}_{(x, y, z)}$,

where

$\mathbf{c}=\left[\begin{array}{ccccc}c_{11} & c_{12} & 0 & 0 & c_{16} \\ c_{12} & c_{22} & 0 & 0 & c_{26} \\ 0 & 0 & c_{44} & c_{45} & 0 \\ 0 & 0 & c_{45} & c_{55} & 0 \\ c_{16} & c_{26} & 0 & 0 & c_{66}\end{array}\right]$

with

$$
\begin{aligned}
& c_{11}=8-\alpha_{2}(1-\cos 2 \theta)-\alpha_{3}(1-\cos 4 \theta), \\
& c_{12}=\alpha_{4}-\alpha_{3} \cos 4 \theta, \\
& c_{16}=\frac{1}{2} \alpha_{2} \sin 2 \theta+\alpha_{3} \sin 4 \theta, \\
& c_{22}=8-\alpha_{2}(1+\cos 2 \theta)-\alpha_{3}(1-\cos 4 \theta), \\
& c_{26}=\frac{1}{2} \alpha_{2} \sin 2 \theta-\alpha_{3} \sin 4 \theta, \\
& c_{44}=\alpha_{8}-\alpha_{9} \cos 2 \theta, \\
& c_{45}=\alpha_{9} \sin 2 \theta, \\
& c_{55}=\alpha_{8}+\alpha_{9} \cos 2 \theta, \\
& c_{66}=\frac{1}{2}\left(8-\alpha_{2}-\alpha_{3}-\alpha_{4}\right)-\alpha_{3} \cos 4 \theta .
\end{aligned}
$$

Assuming small displacements and using Hamilton's variational principle along with an eight node isoparametric plate element with nine degrees of freedom per node, corresponding to the nine expansion terms in the displacement equation (1), we obtain the following equilibrium equation for free harmonic vibrations at the element level:

$\mathbf{K}^{e} \mathbf{a}_{i}^{e}-\lambda_{i}^{e} \mathbf{M}^{e} \mathbf{a}_{i}^{e}=\mathbf{0}$,

where $\mathbf{K}^{e}$ and $\mathbf{M}^{e}$ are the element stiffness and mass matrices, respectively, and $\mathbf{a}_{i}^{e}$ are the eigenvectors associated to the eigenvalues $\lambda_{i}^{e}$, at the element level. Second degree serendipity shape functions were used to interpolate the displacement field within an element [22].

The equilibrium equation for the whole discretised plate assumed free in space is therefore [23]

$(\mathbf{K}+\beta \mathbf{M}) \mathbf{a}_{i}-\left(\lambda_{i}+\beta\right) \mathbf{M} \mathbf{a}_{i}=\mathbf{0}$,

where $\mathbf{K}$ and $\mathbf{M}$ are the stiffness and mass matrices of the plate, and $\mathbf{a}_{i}$ the eigenvector associated to the numerical eigenvalue $\lambda_{i}$. In order for the stiffness matrix to be positive definite, a shift $\beta \mathbf{M}$ is applied to it (usually $\beta$ is of the same magnitude as the first nonzero eigenvalue).

\section{Experimental method}

The experimental eigenfrequencies of a completely free plate are obtained using the experimental setup shown in Fig. 3.

In practice a truly free support cannot be provided. However, a suspension which closely approximates this condition can be achieved by supporting the test plate on light elastic bands so that the rigid body modes have very low natural frequencies in relation to those of the bending modes. These elastic bands were simply glued to the plate edge (approximately at the mid points of two neighbour edges).

The simplest and fastest way to excite the vibration modes of a plate is the impulse technique, so a hand held hammer was used to impact the plate and the force transducer attached to the hammer head measures the input force.

A condenser microphone was used to measure the plate response. The microphone is placed in a small table stand perpendicular to the plate.

The analog signals from the impact hammer and microphone were fed into a dual channel signal analyser. Both signals are low-pass filtered, sampled and subjected to appropriate time weighting functions. The weighting is important in order to reduce noise and avoid a leakage error caused by the truncation of the sampled time signal. The signals are fast Fourier transformed and divided to obtain the frequency response function (FRF). All of these steps are handled by the signal analyser.

The digital process described above results in a discrete spectrum which contains a finite number of values for the FRF. The FRF is generally a complex valued function and a peak usually indicates the presence of a natural frequency, however, the frequency of maximum response is not an accurate estimate of the natural frequency. First of all, the spectrum is discrete with a certain resolution and the peak value may not rely entirely on a single point. The second most serious reason is that neighbouring modes contribute a certain amount to the total response at the resonance of the mode being analysed. Finally, the third reason is that the values of the FRF include damping, which affects slightly the resonant frequencies. To deal with these problems,

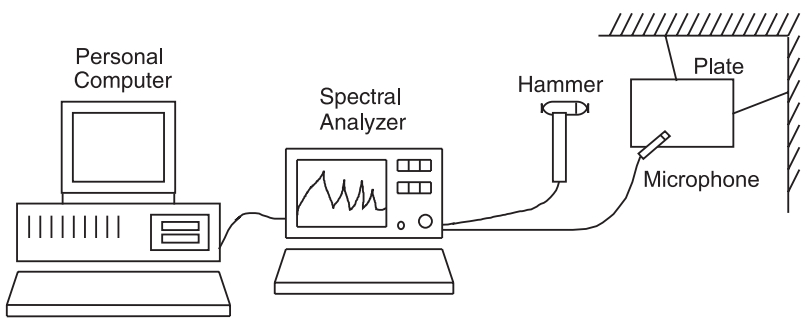

Fig. 3. Schematic representation of experimental setup. 
refined modal analysis methods have been developed. In this work the rational fraction polynomial (RFP) method as described in [24] was used for the curve fitting process on a personal computer suited with an interface card.

\section{The identification method}

The problem of identifying mechanical properties of composite plate specimens involves the comparison of the experimental results described in the previous section with the results produced by the numerical method. In a laminate made out of $M$ different materials the mechanical properties that are to be identified for material $i$ are $E_{1}^{i}, E_{2}^{i}, G_{12}^{i}, v_{12}^{i}, G_{23}^{i}$ and $G_{13}^{i}$. The experimental results that are used in this process are the first $I$ natural frequencies of the free plate.

This problem is solved using optimisation techniques by minimising an error estimator $\boldsymbol{\Phi}$, which expresses the difference between the response of the numerical model and the corresponding experimental results. In Fig. 4, a schematic representation of the identification method is presented, where index $k$ represents the current iteration and $\mathbf{r}_{\mathrm{e}}$ represents the experimental response:

$\mathbf{r}_{\mathrm{e}}=\left\{\tilde{\lambda}_{1}, \ldots, \tilde{\lambda}_{I}\right\}^{\mathrm{T}}$,

where $\tilde{\lambda}_{i}=\tilde{\omega}_{i}^{2}$ are the experimental eigenvalues and $I$ is the total number of measured experimental eigenfrequencies.

The response of the numerical model is $\mathbf{f}(\mathbf{p})=\left\{\lambda_{1}, \ldots, \lambda_{I}\right\}^{\mathrm{T}}$, where $\lambda_{i}=\omega_{i}^{2}$ are the eigenvalues obtained through this model and $p$ is the vector of design variables:

$\mathbf{p}=\left\{\bar{\alpha}^{1^{\mathrm{T}}}, \ldots, \bar{\alpha}^{M^{\mathrm{T}}}\right\}^{\mathrm{T}}$,

in which $\bar{\alpha}^{i}$ contains the non-dimensional material parameters for material $i$,

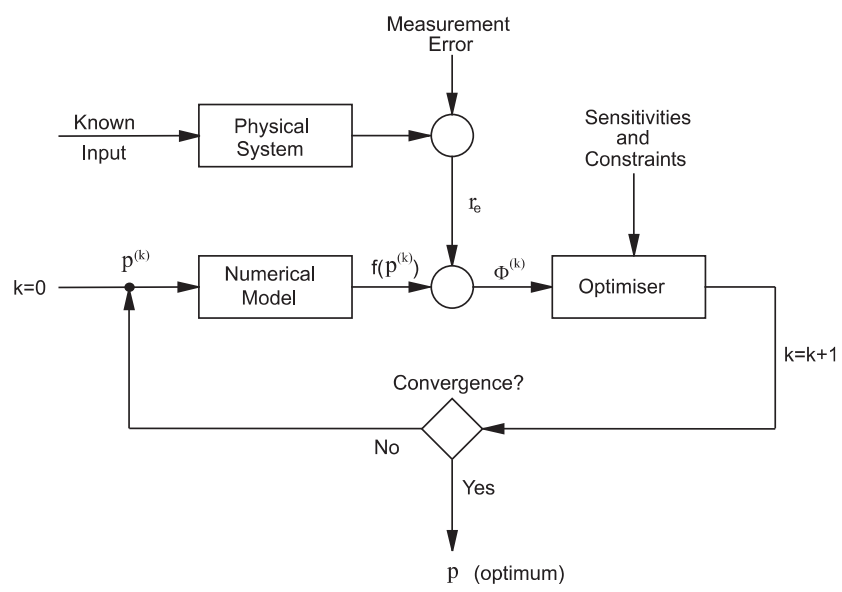

Fig. 4. Schematic representation of the identification method. $\bar{\alpha}^{i}=\left\{\bar{\alpha}_{1}^{i}, \ldots, \bar{\alpha}_{6}^{i}\right\}^{\mathrm{T}}$.

The non-dimensional material parameters for material $i$ are defined using Eq. (4):

$$
\begin{aligned}
& \bar{\alpha}_{1}^{i}=E_{1}^{i} /{ }^{0} E_{1}^{i}, \\
& \bar{\alpha}_{2}^{i}=\alpha_{2}^{i}, \\
& \bar{\alpha}_{3}^{i}=\alpha_{3}^{i}, \\
& \bar{\alpha}_{4}^{i}=\alpha_{4}^{i}, \\
& \bar{\alpha}_{5}^{i}=\alpha_{8}^{i}, \\
& \bar{\alpha}_{6}^{i}=\alpha_{9}^{i} .
\end{aligned}
$$

It should be noted that, in Eq. (14), the superscript $i$ was added to the non-dimensional parameters of Eq. (4) with the purpose of referencing the different materials in the laminate. In Eq. (14), ${ }^{0} E_{1}^{i}$ is the initial guess for the longitudinal Young's modulus of material $i$.

\subsection{Error measure}

In order to establish an effective comparison between experimental results and the response of the numerical model it is necessary to define an error measure that, in general, can be of the following form [25]:

$$
\boldsymbol{\Phi}=\left(\mathbf{r}_{\mathrm{e}}-\mathbf{f}(\mathbf{p})\right)^{\mathrm{T}} \mathbf{W}_{\mathbf{r}}\left(\mathbf{r}_{\mathrm{e}}-\mathbf{f}(\mathbf{p})\right)+\left(\mathbf{p}^{(0)}-\mathbf{p}\right)^{\mathrm{T}} \mathbf{W}_{\mathbf{p}}\left(\mathbf{p}^{(0)}-\mathbf{p}\right) .
$$

The weighting matrix $\mathbf{W}_{\mathbf{r}}$ expresses the confidence on the measured natural frequencies while the weighting matrix $\mathbf{W}_{\mathbf{p}}$ takes into consideration the reliability of the initial estimate $\mathbf{p}^{(0)}$. Of the two terms in Eq. (15), the first one represents the gap between the model response and the experimental one and the second one represents the deviation between the initial guess for the non-dimensional parameters and the real parameters that are to be determined. Each one of these terms is an L2 error estimator, weighted by $\mathbf{W}_{\mathbf{r}}$ and $\mathbf{W}_{\mathbf{p}}$.

For the sake of simplicity it was decided to use only the first term of Eq. (15), with $\mathbf{W}_{\mathbf{p}}=\mathbf{0}$ and with the following response weighting matrix:

$\mathbf{W}_{\mathbf{r}}=\left[\begin{array}{ccc}w_{1} / \tilde{\lambda}_{1}^{2} & & 0 \\ & \ddots & \\ 0 & & w_{I} / \tilde{\lambda}_{I}^{2}\end{array}\right]$,

in which $w_{i} \in[0,1]$ are weighting factors that express the confidence level in each eigenfrequency $i$, the error measure in Eq. (15) can be rewritten as

$\boldsymbol{\Phi}=\sum_{i=1}^{I} \mathbf{w}_{i}\left(\frac{\tilde{\lambda}_{i}-\lambda_{i}}{\tilde{\lambda}_{i}}\right)^{2}$.

Eq. (17) can easily be identified as a weighted leastsquares error estimator. 


\subsection{The minimisation problem}

The problem of identifying mechanical properties of composite materials can be formulated as a minimisation problem:

$$
\begin{aligned}
\min & \boldsymbol{\Phi}(\mathbf{p}) \geqslant 0 \\
\text { s.t. } & \mathbf{g}(\mathbf{p}) \leqslant \mathbf{0}, \\
& \mathbf{p}^{l} \leqslant \mathbf{p} \leqslant \mathbf{p}^{u},
\end{aligned}
$$

where $\mathbf{p}^{l}$ and $\mathbf{p}^{u}$ are the side constraints on the design variables $p, g$ is the vector of the imposed constraints on the design variables in order for the constitutive matrix for each material in Eq. (3) to be positive definite [5,21],

$\mathbf{g}=\left\{\mathbf{g}^{1^{\mathrm{T}}}, \ldots, \mathbf{g}^{M^{\mathrm{T}}}\right\}^{\mathrm{T}}$,

in which $\mathbf{g}^{i}$ are the vectors that contain the six constraints associated with material $i$,

$\mathbf{g}^{i}=\left\{g_{1}^{i}, \ldots, g_{6}^{i}\right\}^{\mathrm{T}}$

with the following components:

$$
\begin{aligned}
& g_{1}^{i}=-\bar{\alpha}_{1}^{i}, \\
& g_{2}^{i}=\frac{\bar{\alpha}_{2}^{i}}{\bar{\alpha}_{2}^{i}-4}, \\
& g_{3}^{i}=\frac{8-\bar{\alpha}_{2}^{i}-3 \alpha_{3}^{i}-\bar{\alpha}_{4}^{i}}{-16 \bar{\alpha}_{0}^{i}}, \\
& g_{4}^{i}=\left|\frac{\bar{\alpha}_{4}^{i}-\bar{\alpha}_{3}^{i}}{8-2 \bar{\alpha}_{2}^{i}}\right|-\sqrt{\frac{4}{4-\bar{\alpha}_{2}^{i}}}, \\
& g_{5}^{i}=\frac{\bar{\alpha}_{5}^{i}-\bar{\alpha}_{6}^{i}}{-8 \bar{\alpha}_{0}^{i}}, \\
& g_{6}^{i}=\frac{\bar{\alpha}_{5}^{i}+\bar{\alpha}_{6}^{i}}{-8 \bar{\alpha}_{0}^{i}},
\end{aligned}
$$

which can be expressed in terms of the physical constants:

$$
\begin{aligned}
& g_{1}^{i}=-\frac{E_{1}^{i}}{{ }^{0} E_{1}^{i}}, \\
& g_{2}^{i}=E_{2}^{i}-E_{1}^{i}, \\
& g_{3}^{i}=-\frac{G_{12}^{i}}{E_{1}^{i}}, \\
& g_{4}^{i}=\left|v_{12}^{i}\right|-\sqrt{E_{1}^{i} / E_{2}^{i}}, \\
& g_{5}^{i}=-\frac{G_{23}^{i}}{E_{1}^{i}}, \\
& g_{6}^{i}=-\frac{G_{13}^{i}}{E_{1}^{i}} .
\end{aligned}
$$

The minimisation problem formulated above is solved using non-linear mathematical programming techniques. To carry out the constrained minimisation $a$ feasible directions non-linear interior point algorithm developed by Herskovits [26] is used. The choice of this algorithm was made by one very important reason: the objective function cannot be defined at infeasible points, since the stiffness matrix is only positive definite at interior points.

\subsection{Sensitivity analysis}

The minimisation described in the previous section demands the sensitivities of the objective function $\boldsymbol{\Phi}$. Sensitivity analysis consists in determining, for a given perturbation $\delta \mathbf{p}$ in the design variables, the variation $\delta \boldsymbol{\Phi}$ of the objective function:

$\delta \boldsymbol{\Phi}=\frac{\partial \Phi}{\partial \mathbf{p}} \cdot \delta \mathbf{p}$.

If we differentiate Eq. (17) with respect to $\mathbf{p}$ we obtain

$\frac{\partial \boldsymbol{\Phi}}{\partial \mathbf{p}}=-2 \sum_{i=1}^{I} \mathbf{w}_{i}\left(1-\frac{\lambda_{i}}{\tilde{\lambda}_{i}}\right) \frac{1}{\tilde{\lambda}_{i}} \frac{\partial \lambda_{i}}{\partial \mathbf{p}}$.

The problem of sensitivity evaluation is therefore reduced to the evaluation of the derivatives of the eigenvalues $\lambda_{i}$ in order to design variables p. From the equilibrium equation (10) (with $\beta=0$ ) it is possible to obtain the components of $\partial \lambda_{i} / \partial \mathbf{p}$ (with the eigenvectors $\mathbf{a}_{i}$ mass-normalised):

$\frac{\partial \lambda_{i}}{\partial p_{j}}=\mathbf{a}_{i}^{\mathrm{T}}\left(\frac{\partial \mathbf{K}}{\partial p_{j}}-\lambda_{i} \frac{\partial \mathbf{M}}{\partial p_{j}}\right) \mathbf{a}_{i}$.

In the present case the mass matrix does not depend on the design variables, hence

$\frac{\partial \lambda_{i}}{\partial p_{j}}=\mathbf{a}_{i}^{\mathrm{T}} \frac{\partial \mathbf{K}}{\partial p_{j}} \mathbf{a}_{i}$.

It is desirable, for the sake of computational efficiency, to evaluate Eq. (26) at the element level, adding the contribution of all the elements:

$\frac{\partial \lambda_{i}}{\partial p_{j}}=\sum_{e=1}^{\mathrm{NE}} \mathbf{a}_{i}^{e^{\mathrm{T}}} \frac{\partial \mathbf{K}^{e}}{\partial p_{j}} \mathbf{a}_{i}^{e}$,

where NE is the total number of elements in which the plate is discretised.

The evaluation of the derivatives of the element stiffness matrix is performed analytically, considering that only the elasticity matrices depend on the design variables.

Results showing the quality of sensitivities for a plate made up of two different materials have been published by Araújo et al. [27]. These results show that the technique described here can be applied with confidence in the optimisation of material parameters of composite materials. 
Table 1

Experimentally measured frequencies $(\mathrm{Hz})$ and residuals (\%) obtained after identification for specimens in Example 1

\begin{tabular}{|c|c|c|c|c|c|c|c|c|c|c|c|c|c|c|}
\hline & $I$ & 1 & 2 & 3 & 4 & 5 & 6 & 7 & 8 & 9 & 10 & 11 & 12 & 13 \\
\hline \multirow[t]{2}{*}{ S1.1 } & $\tilde{\omega}_{i}$ & 125.05 & 203.50 & 331.30 & 446.65 & 493.55 & 603.80 & 650.75 & 818.70 & 951.35 & 990.65 & 1256.75 & 1343.80 & - \\
\hline & $r_{i}$ & 0.09 & -0.05 & 0.14 & 0.15 & -0.07 & 0.06 & 0.06 & -0.14 & -0.10 & -0.11 & 0.00 & 0.14 & - \\
\hline \multirow[t]{2}{*}{$\mathrm{S} 1.2$} & $\tilde{\omega}_{i}$ & 167.70 & 198.65 & 414.20 & 480.70 & - & 633.90 & 738.50 & 909.20 & 1016.5 & 1176.3 & 1300.60 & 1388.70 & 1418.70 \\
\hline & $r_{i}$ & -0.03 & -0.07 & 0.02 & 0.02 & - & 0.07 & 0.02 & 0.10 & -0.07 & 0.03 & -0.01 & -0.10 & 0.02 \\
\hline \multirow[t]{2}{*}{ S1.3 } & $\tilde{\omega}_{i}$ & 129.85 & 218.70 & - & 395.10 & 459.75 & 630.45 & 722.20 & 878.50 & 1000.20 & 1026.50 & 1167.40 & 1261.05 & 1402.00 \\
\hline & $r_{i}$ & 0.07 & -0.28 & - & 0.24 & 0.45 & -0.07 & 0.50 & -0.53 & -0.07 & 0.19 & -0.03 & 0.25 & -0.57 \\
\hline \multirow[t]{2}{*}{ S1.4 } & $\tilde{\omega}_{i}$ & 137.65 & 221.50 & 403.05 & 430.75 & 472.90 & 642.45 & 758.30 & 879.50 & 1035.30 & 1119.40 & 1250.30 & 1340.00 & - \\
\hline & $r_{i}$ & 0.01 & -0.01 & 0.02 & -0.03 & -0.04 & 0.12 & 0.03 & -0.04 & -0.02 & -0.04 & -0.03 & 0.03 & - \\
\hline
\end{tabular}

\section{Applications}

The following examples illustrate the application of the method described in the previous sections. For each example the experimental eigenfreqencies are presented, as well as the corresponding percentage residuals at the optimum:

$r_{i}=\frac{\tilde{\omega}_{i}-\omega_{i}}{\tilde{\omega}_{i}} \times 100$.

Each identification set of results is compared with strain gauge measurements.

It is assumed that weight factors $\mathbf{w}_{i}=1$ were used in all examples, except otherwise specified. A $12 \times 12$ finite element mesh is used for all the examples.

Example 1. Four IM7 carbon fibre reinforced epoxy resin 977-2 (Fiberite) plate specimens (S), made of unidirectional fibres with a nominal thickness of $0.135 \mathrm{~mm}$ for $60 \% V_{\mathrm{f}}$, with stacking sequences

- $\left[45^{\circ}{ }_{3}, 0^{\circ}{ }_{3},-45^{\circ}, 90^{\circ}\right]_{\mathrm{s}}(\mathrm{S} 1.1)$,

- $\left[45^{\circ}, 0^{\circ},-45^{\circ}, 90^{\circ}, 45^{\circ}{ }_{2}, 0^{\circ}{ }_{2},-45^{\circ}{ }_{2}, 90^{\circ}{ }_{2}\right]_{\mathrm{S}}(\mathrm{S} 1.2)$,

- $\left[45^{\circ},-45^{\circ}, 0^{\circ}{ }_{3}, 90^{\circ}\right]_{\mathrm{s}}(\mathrm{S} 1.3)$ and

- $\left[45^{\circ},-45^{\circ}{ }_{3}, 0^{\circ}{ }_{5}, 90^{\circ}\right]_{\mathrm{s}}(\mathrm{S} 1.4)$

were studied. The specimens dimensions and masses are:

- S1.1: $a=210.5 \mathrm{~mm}, b=311 \mathrm{~mm}, h=3.06 \mathrm{~mm}$, $m=0.3225 \mathrm{~kg}$

- S1.2: $a=210.5 \mathrm{~mm}, b=311 \mathrm{~mm}, h=3.07 \mathrm{~mm}$, $m=0.3232 \mathrm{~kg}$;

- S1.3: $a=210 \mathrm{~mm}, b=311 \mathrm{~mm}, h=3.04 \mathrm{~mm}, m=$ $0.320 \mathrm{~kg}$;

- S1.4: $a=210.5 \mathrm{~mm}, b=310.5 \mathrm{~mm}, h=3.09 \mathrm{~mm}$, $m=0.3252 \mathrm{~kg}$.

The initial guess for the elastic constants corresponds to a typical unidirectional layer of carbon fibre reinforced epoxy resin with $50 \% V_{\mathrm{f}}$ :

${ }^{0} E_{1}=117.2 \mathrm{GPa}, \quad{ }^{0} E_{2}=8.8 \mathrm{GPa}$,

${ }^{0} G_{12}={ }^{0} G_{23}={ }^{0} G_{13}=3.1 \mathrm{GPa}, \quad{ }^{0} v_{12}=0.35$.

In Table 1 it can be seen that, for specimens S1.2 and $\mathrm{S} 1.3$, there are some experimental frequencies that were not detected. So, the weighting factor associated with these were $\mathbf{w}_{i}=0$.

Identification results are presented in Table 2 along with strain gauge test results on specimens with unidirectional layers made of the same material. All the identified properties are in good agreement with the tensile test results, except for the major Poisson's ratio $v_{12}$, whose values present a pronounced oscillation and do not agree satisfactorily with the strain gauge test results.

Example 2. Two E glass fibre reinforced epoxy resin R368 (Structil) plate specimens (S), made of unidirectional fibres with a nominal thickness of $0.130 \mathrm{~mm}$ for $60 \% V_{\mathrm{f}}$, with stacking sequences $\left[\left(0^{\circ}, 90^{\circ}\right)_{2}, 90^{\circ}, 0^{\circ}, 90^{\circ}\right]_{\mathrm{s}}$ (S2.1) and $\left[0^{\circ}{ }_{2}, 90^{\circ}, 0^{\circ}{ }_{2}, 90^{\circ}\right]_{\mathrm{S}}(\mathrm{S} 2.2)$ were studied. The specimens dimensions and masses are:

- S2.1: $a=191 \mathrm{~mm}, b=283 \mathrm{~mm}, h=2.89 \mathrm{~mm}, m=$ $0.2571 \mathrm{~kg}$;

- S2.2: $a=191 \mathrm{~mm}, b=282 \mathrm{~mm}, h=2.87 \mathrm{~mm}, m=$ $0.2511 \mathrm{~kg}$.

The initial guess for the elastic constants corresponds to a typical unidirectional layer of E glass reinforced epoxy resin with $50 \% V_{\mathrm{f}}$ :

${ }^{0} E_{1}=45 \mathrm{GPa}, \quad{ }^{0} E_{2}=4.5 \mathrm{GPa}$,

${ }^{0} G_{12}={ }^{0} G_{23}={ }^{0} G_{13}=3.7 \mathrm{GPa}, \quad{ }^{0} v_{12}=0.28$.

Identification results are presented in Table 3. Some tensile test results are presented and compared with

Table 2

Identified properties and strain gauge results for Example 1 (modulus in $\mathrm{GPa})^{\mathrm{a}}$

\begin{tabular}{lccccl}
\hline Specimen & S1.1 & S1.2 & S1.3 & S1.4 & $\begin{array}{l}\text { Strain } \\
\text { gauge }\end{array}$ \\
\hline$E_{1}$ & 161.1 & 162.3 & 164.8 & 159.6 & $\begin{array}{l}170 \pm 1^{(\mathrm{T})} \\
150 \pm 5^{(\mathrm{C})}\end{array}$ \\
$E_{2}$ & 9.3 & 8.9 & 9.6 & 9.8 & $10 \pm 1^{(\mathrm{T})}$ \\
$G_{12}$ & 6.8 & 6.0 & 6.8 & 6.2 & 5.8 \\
$G_{13}$ & 3.1 & 2.4 & 2.6 & 3.0 & - \\
$G_{23}$ & 3.2 & 2.4 & 1.4 & 4.4 & - \\
$v_{12}$ & 0.166 & 0.249 & -0.036 & 0.264 & 0.35 \\
\hline
\end{tabular}

$\mathrm{a}(\mathrm{T})-$ Tension; ${ }^{(\mathrm{C})}-$ compression 
Table 3

Identified properties for Example 2 (modulus in GPa)

\begin{tabular}{lcc}
\hline Specimen & S2.1 & S2.2 \\
\hline$E_{1}$ & 28.8 & 29.4 \\
$E_{2}$ & 8.6 & 7.2 \\
$G_{12}$ & 2.7 & 2.7 \\
$G_{13}$ & 3.9 & 1.3 \\
$G_{23}$ & 3.9 & 1.3 \\
$v_{12}$ & 0.287 & 0.407 \\
\hline
\end{tabular}

Table 4

Comparison between identified and tensile global $E_{y}(\mathrm{GPa})$ for Example 2

\begin{tabular}{lll}
\hline Specimen & Identified & Tensile test \\
\hline S2.1 & 20.3 & 22.5 \\
S2.2 & 17.0 & 17.5 \\
\hline
\end{tabular}

global identified results in Table 4. The global identified mechanical properties are obtained using the procedure described in Appendix A.

The values obtained in this example for the mechanical properties are in relatively good agreement with each other, except for the major Poisson's ratio $v_{12}$ and the transverse shear modulus $G_{13}$ and $G_{23}$. A good agreement is obtained between the global identified and the tensile test results, with very low residual level as shown in Table 5 .

Example 3. A mixed glass/carbon (g/c) fibre reinforced epoxy plate specimen with plies made of unidirectional fibres, respectively, of E glass in a R368 resin (Structil) with a nominal thickness of $0.130 \mathrm{~mm}$ for $60 \% V_{\mathrm{f}}$ and T300 carbon fiber in an epoxy resin R367 (Structil) with a nominal thickness of $0.327 \mathrm{~mm}$ for $60 \% V_{\mathrm{f}}$, with a stacking sequence $\left[0^{\circ}, 90_{\mathrm{g}}^{\circ}, 0^{\circ}, 90^{\circ}{ }_{2 \mathrm{~g}}, 0^{\circ}{ }_{\mathrm{c}}, 90_{\mathrm{g}}^{\circ}\right]_{\mathrm{s}}$ was studied. The specimen dimensions and mass are:

- $a=192.5 \mathrm{~mm}, b=292 \mathrm{~mm}, h=3.83 \mathrm{~mm}, m=$ $0.3369 \mathrm{~kg}$.

The prepregs used to build these specimens are: Structil $200 \mathrm{~g} / \mathrm{m}^{2} \mathrm{VEE}_{2} 20 \mathrm{R} 368$ for glass plies and Structil $350 \mathrm{~g} /$ $\mathrm{m}^{2} \mathrm{CTE}_{2} 35 \mathrm{R} 367$ for carbon plies. Hence, the initial guess for the elastic constants of the glass plies corresponds to a typical layer of this unidirectional glass with $50 \% V_{\mathrm{f}}$ :

${ }^{0} E_{1}=45 \mathrm{GPa}, \quad{ }^{0} E_{2}=4.5 \mathrm{GPa}$,

${ }^{0} G_{12}={ }^{0} G_{23}={ }^{0} G_{13}=3.7 \mathrm{GPa}, \quad{ }^{0} v_{12}=0.28$, while the initial guess for the elastic constants of the carbon plies corresponds to a typical layer of this unidirectional carbon with $50 \% V_{\mathrm{f}}$ :

$$
\begin{aligned}
& { }^{0} E_{1}=117.2 \mathrm{GPa}, \quad{ }^{0} E_{2}=8.8 \mathrm{GPa} \\
& { }^{0} G_{12}={ }^{0} G_{23}={ }^{0} G_{13}=3.1 \mathrm{GPa}, \quad{ }^{0} v_{12}=0.35 .
\end{aligned}
$$

The first step was to obtain the global properties of the specimen, using in the numerical model only one equivalent single layer (ESL).

The second step was to calculate the fibre volume for carbon and glass plies as well as the epoxy matrix volume, using the nominal and actual thickness of the specimen and data available from Structil. With the obtained data it is then possible to estimate the correct thickness for each material ply, as well as relative densities, based on the assumption that the epoxy matrix distribution is proportional to the nominal thickness of each layer.

Finally a complete model (CM) of the plate specimen was used in order to identify the properties of each material layer.

Results for global properties using the ESL and the $\mathrm{CM}$ are presented in Table 6, while individual layer results are presented in Table 7.

The measured natural frequencies and residuals obtained after identification for the two described situations are presented in Table 8.

It can be seen that the identified $E_{x}$ for the ESL model is quite good when compared with the equivalent one determined through tensile testing of a specimen using a strain gauge. Concerning the results for the CM, the values of the global $E_{x}$, which were calculated using the identified properties for each layer in accordance with the procedure described in Appendix A, present a worst agreement with the tensile test results than those obtained through the global model (ESL). We think that this is due to the uncertainties in determining the correct thickness, and consequently the matrix and fibre volume distribution of the glass and carbon plies on hybrid composite panels, that affect the ply elastic properties and density.

Although one could conclude that the number of experimental natural frequencies is not enough for the 12 design variables involved, it can be seen in Fig. 5 that this might not be the case.

Table 5

\begin{tabular}{|c|c|c|c|c|c|c|c|c|c|c|c|c|c|}
\hline & $I$ & 1 & 2 & 3 & 4 & 5 & 6 & 7 & 8 & 9 & 10 & 11 & 12 \\
\hline \multirow[t]{2}{*}{$\mathrm{S} 2.1$} & $\tilde{\omega}_{i}$ & 72.78 & 121.00 & 191.38 & 286.78 & 315.12 & 340.40 & 400.12 & 433.84 & 634.81 & 653.56 & 712.43 & 789.97 \\
\hline & $r_{i}$ & 0.27 & -0.24 & 0.04 & 0.04 & -0.04 & 0.11 & -0.08 & -0.25 & -0.35 & 0.30 & 0.03 & 0.15 \\
\hline \multirow[t]{2}{*}{$\mathrm{S} 2.2$} & $\tilde{\omega}_{i}$ & 72.22 & 108.57 & 182.73 & 293.94 & 308.74 & 343.48 & 369.41 & 442.62 & 586.17 & 623.61 & 649.79 & 845.90 \\
\hline & $r_{i}$ & 0.08 & -0.48 & 0.00 & 0.32 & -0.10 & 0.30 & 0.18 & -0.23 & 0.13 & -0.20 & -0.04 & 0.02 \\
\hline
\end{tabular}

Experimentally measured frequencies $(\mathrm{Hz})$ and residuals (\%) obtained after identification for specimens in Example 2 
Table 6

Identified global properties using ESL, CM and strain gauge results for Example 3 (modulus in GPa)

\begin{tabular}{llll}
\hline & $\begin{array}{l}\text { Identified } \\
(\mathrm{ESL})\end{array}$ & $\begin{array}{l}\text { Identified } \\
(\mathrm{CM})\end{array}$ & Strain gauge \\
\hline$E_{x}$ & 76.2 & 69.7 & 77.0 \\
$E_{y}$ & 15.3 & 20.7 & - \\
$G_{x y}$ & 3.7 & 4.0 & - \\
$G_{x z}$ & 1.8 & 1.8 & - \\
$G_{y z}$ & 3.3 & 3.4 & - \\
$v_{x y}$ & 0.183 & 0.132 & $0.15-0.17$ \\
\hline
\end{tabular}

Table 7

Identified properties (CM) in Example 3 (modulus in GPa)

\begin{tabular}{lcc}
\hline Ply type & Glass & Carbon \\
\hline$E_{1}$ & 43.1 & 103.3 \\
$E_{2}$ & 4.6 & 8.7 \\
$G_{12}$ & 3.8 & 4.1 \\
$G_{13}$ & 3.7 & 1.0 \\
$G_{23}$ & 3.4 & 3.2 \\
$v_{12}$ & 0.280 & 0.402 \\
\hline
\end{tabular}

Table 8

Experimentally measured frequencies and residuals obtained after identification through ESL and CM for Example 3

\begin{tabular}{rrrr}
\hline \multicolumn{1}{c}{$i$} & $\tilde{\omega}_{i}(\mathrm{~Hz})$ & $r_{i}(\mathrm{ESL} ; \%)$ & $r_{i}(\mathrm{CM} ; \%)$ \\
\hline 1 & 112.40 & 0.02 & 0.01 \\
2 & 316.25 & 0.05 & 0.05 \\
3 & 337.75 & -0.13 & -0.13 \\
4 & 392.70 & -0.01 & -0.01 \\
5 & 401.05 & -0.08 & -0.08 \\
6 & 650.85 & 0.04 & 0.04 \\
7 & 881.90 & -0.00 & -0.01 \\
8 & 907.75 & 0.19 & 0.19 \\
9 & 933.45 & -0.27 & -0.27 \\
10 & 977.95 & 0.00 & 0.00 \\
11 & 1160.15 & 0.07 & 0.07 \\
12 & 1177.30 & 0.15 & 0.15 \\
13 & 1623.80 & -0.02 & -0.02 \\
14 & 1713.55 & -0.21 & -0.21 \\
15 & 1754.30 & 0.61 & 0.61 \\
16 & 1775.40 & -0.40 & -0.40 \\
17 & 1814.45 & 0.22 & 0.22 \\
18 & 1947.54 & -0.14 & -0.13 \\
19 & 2007.40 & 0.21 & 0.21 \\
20 & 2356.90 & -0.34 & -0.34 \\
\hline
\end{tabular}

\section{Conclusions}

A non-destructive numerical-experimental method for the identification of up to six elastic constants per material on composite plate specimens made up of different materials has been discussed. The method is based on a numerical higher-order finite element model, using analytical sensitivities, as well as on experimental nondestructive free vibration analysis.

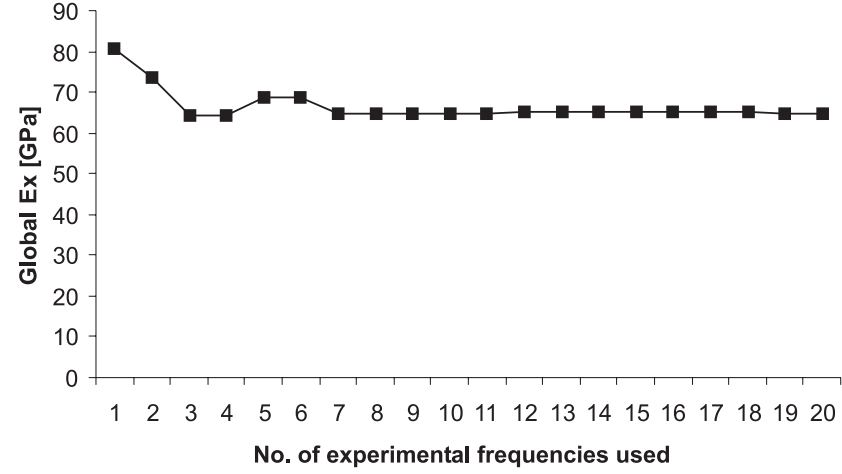

Fig. 5. Example 3, complete model: graph showing the dependence of global $E_{x}$ with respect to the number of experimental frequencies used.

The mechanical properties evaluated through this technique are valid on average for the entire specimen, whereas the classical tensile test measurements are only valid in a specific point of the test specimen.

The mechanical properties $E_{1}, E_{2}$ and $G_{12}$ are always evaluated without major discrepancies in single-material plates.

As for the transverse shear modulus $G_{13}$ and $G_{23}$, the plates tested in this work are not thick enough for them to be correctly identified. Thus, the identified values of the transverse shear modulus can be disregarded as the sensitivity to these modulus is very low. As for the major Poisson's ratio $v_{12}$, some discrepancies are found in the test cases. This can be explained if we consider the results of a sensitivity analysis carried out by Frederiksen [5], according to which the sensitivity of $v_{12}$ is very small when compared to the sensitivities of the other in-plane mechanical properties. This is specially pronounced for the most strongly anisotropic specimens presented, as can be seen in specimen S1.3.

Regarding the identification of mechanical properties in composite plates made up of layers with different materials, more tests are required in order to explain the discrepancies found.

Finally, the non-linear interior point algorithm used in the optimisation phase proved to be very robust and efficient. The CPU times involved in the overall process of identification are of the order of 10-20 min using a personal computer equiped with a $450 \mathrm{MHz}$ Pentium II processor and $128 \mathrm{MB}$ RAM.

\section{Acknowledgements}

The authors thank Fundação para a Ciência e Tecnologia (FCT) for the financial support, through project PRAXIS/P/EME/12028/1998 and FCT/ICCTI (Portugal) and CNPq (Brazil). 


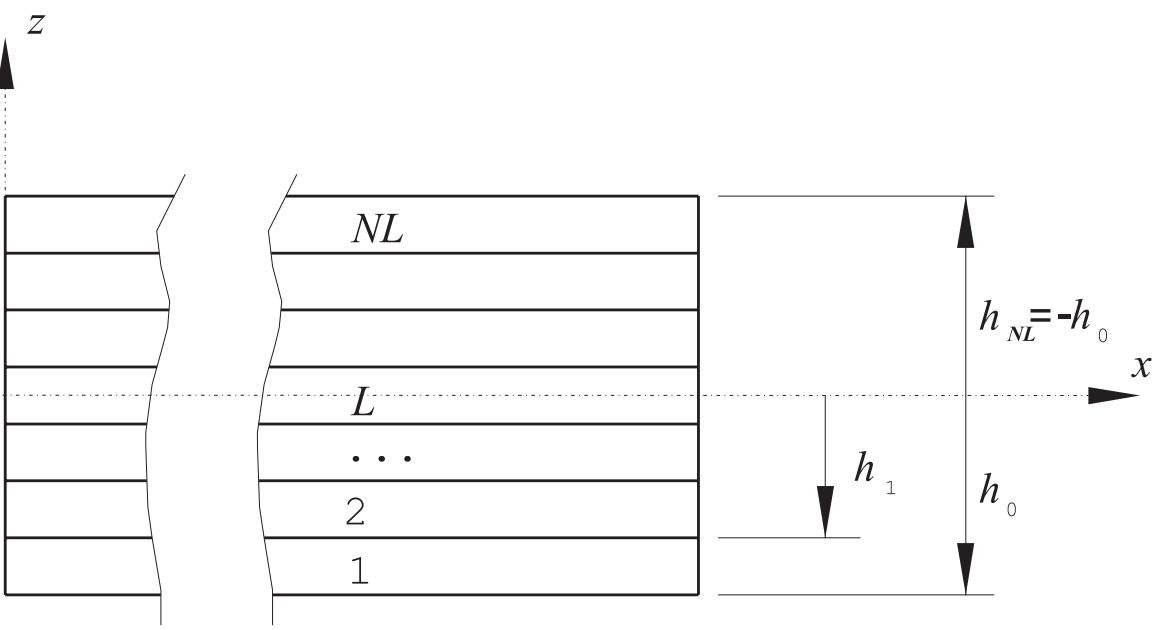

Fig. 6. Layer numbering used for a typical laminate.

\section{Appendix A}

The global properties of a laminate, in the $(x, y, z)$ coordinate system, can be obtained using the procedure described herein $[19,21]$.

Let $\mathbf{C}$ be the matrix that relates membrane and transverse shear forces with the corresponding membrane deformations and distortions:

$\mathbf{C}=\sum_{L=1}^{\mathrm{NL}}\left(h_{L}-h_{L-1}\right)\left(\frac{E_{1}}{8 \alpha_{0}} \mathbf{c}\right)^{(L)}$,

where $L$ is the layer number, NL the total number of layers and $h_{L}$ can be depicted from Fig. 6. Matrix $\mathbf{c}$ is the same as defined in Eqs. (7) and (8).

By inverting $\mathbf{C}$ and pre-multiplying the inverse by the thickness of the laminate we obtain

$$
\begin{aligned}
\mathbf{S} & =h \mathbf{C}^{-1} \\
& =\left[\begin{array}{ccccc}
1 / E_{x} & -v_{x y} / E_{x} & 0 & 0 & \eta_{x, x y} / G_{x y} \\
-v_{y x} / E_{y} & 1 / E_{y} & 0 & 0 & \eta_{y, x y} / G_{x y} \\
0 & 0 & 1 / G_{y z} & \mu_{y z, x z} / G_{x z} & 0 \\
0 & 0 & \mu_{x z, y z} / G_{y z} & 1 / G_{x z} & 0 \\
\eta_{x y, x} / E_{x} & \eta_{x y, y} / E_{y} & 0 & 0 & 1 / G_{x y}
\end{array}\right],
\end{aligned}
$$

where $E_{x}$ and $E_{y}$ are the global Young modulus in the $x$ and $y$-directions, respectively, $G_{x y}, G_{x z}$ and $G_{y z}$ the transverse shear modulus in planes $x y, x z$ and $y z$, respectively and $v_{x y}$ and $v_{y x}$ are Poisson's ratios in the $x y$ plane. As for the remaining quantities, they are the mutual influence and Chentsov coefficients [21].

\section{References}

[1] Mota Soares CM, Moreira de Freitas M, Araújo AL, Pedersen P Identification of material properties of composite plate specimens. Compos Struct 1993;25:277-85.
[2] Mota Soares CM, Moreira de Freitas MJ, Meira Afonso JM. Identificação de propriedades mecânicas de materiais compósitos utilizando técnicas de optimização. Rev Internac Métod Numér Cálc Diseñ Ingr 1993;9(4):357-73.

[3] Araújo AL, Mota Soares CM, Moreira de Freitas MJ. Characterization of material parameters of composite plate specimens using optimization and experimental vibrational data. Composites: Part B 1996;27B:185-91.

[4] Pedersen P. Optimization method applied to identification of material parameters. In: Eschenauer HA, Thierauf G, editors. Discretization methods and structural optimization - procedures and applications. Berlin: Springer; 1989. p. 277-83.

[5] Frederiksen PS. Identification of material parameters in anisotropic plates - a combined numerical/experimental method. Ph.D. Thesis, Department of Solid Mechanics, The Technical University of Denmark, 1992.

[6] Wilde WP, Sol H. Anisotropic material identification using measured resonant frequencies of rectangular composite plates [vol. 2] Compos Struct 1987;4:2317-24 [London: Elsevier].

[7] Sol H. Identification of the complex moduli of composite materials by a mixed numerical/experimental method. In: Wilde WP, Blain WR, editors. Composite materials design and analysis. Berlin: Springer, 1990. p. 267-79.

[8] Wilde WP. Identification of the rigidities of composite systems by mixed numerical/experimental methods. In: Vautrin A, Sol H, editors. Mechanical identification of composites. Amsterdam: Elsevier; 1991. p. 1-15.

[9] Lai TC, Ip KH. Parameter estimation of orthotropic plates by Bayesian sensitivity analysis. Compos Struct 1996;34:29-42.

[10] Frederiksen PS. Parameter uncertainty and design of optimal experiments for the estimation of elastic constants.. Int. J Solids and Structures 1998;35:1241-60.

[11] Rikards R, Chate A. Identification of elastic properties of composites by method of planning of experiments. Compos Struct 1998:42:257-63.

[12] Rikards R, Chate A, Steinchen W, Kessler A, Bledzki AK. Method for identification of elastic properties of laminates based on experiment design. Composites: Part B 1999;30:279-89.

[13] Bledzki AK, Kessler A, Rikards R, Chate A. Determination of elastic constants of glass/epoxy unidirectional laminates by the vibration testing of plates. Compos Sci Technol 1999;59:2015-24.

[14] Cunha J, Piranda J. The use of model updating techniques in dynamics for identification of stiffness properties of sandwich composite structures. J Braz Soc Mech Scis 1999;XXI(2):313-21 [in Portuguese]. 
[15] Pedersen P. Identification techniques in composite laminates. In: Mota Soares CA, Mota Soares CM, Freitas MJM, editors. Mechanics of composite materials and structures. Dordrecht: Kluwer Academic Publishers; 1999. p. 443-52.

[16] Lo KW, Christensen RM, Wu EM. A higher-order theory for deformations. Part 1: Homogeneous plates. J Appl Mech Trans ASME 1977;44(4):663-8.

[17] Lo KW, Christensen RM, Wu EM. A higher-order theory for deformations. Part 2: Laminated plates. J. Appl Mech 1977;44(2):669-76.

[18] Malikarjuna, Kant T. A critical review and some results of recently developed theories of fibre-reinforced laminated composites and sandwiches. Compos Struct 1993;23:293-312.

[19] Araújo AL. Método numérico/experimental para caracterização mecânica de materiais compósitos. M.Sc. Thesis, Mec. Eng. Dept, IST, Technical University of Lisbon, April 1995 [in Portuguese].

[20] Moita JS, Mota Soares CM, Mota Soares CA. Buckling and dynamic behaviour of laminated composite structures using a discrete higher-order displacement model. Comput Struct 1999;73:407-23.
[21] Jones RM. Mechanics of composite materials. New York: McGraw-Hill; 1975.

[22] Bathe KJ. Finite element procedures in engineering analysis. Englewood Cliffs, NJ: Prentice-Hall; 1982.

[23] Zienkiewicz OC. The finite element method in engineering science. New York: McGraw-Hill; 1971.

[24] Richardson MH, Formenti DL. Parameter estimation from frequency response measurements using rational fraction polynomials. In: Proceedings of the IMAC I. Orlando (FL, USA), Nov 8-10, 1982.

[25] Beck JV, Arnold KJ. Parameter estimation in engineering and science. New York: Wiley; 1977.

[26] Herskovits J. A feasible directions interior point technique for nonlinear optimization. J Optim Theory Appl 1998;99(1):121-46.

[27] Araújo AL, Mota Soares CM, Freitas MJM, Pedersen P. Identification of mechanical properties of composite plate specimens using a discrete higher-order displacement model and experimental vibration data. In: Topping BHV, editor. Advances in analysis and design of composites. Edinburgh: Civil-Comp Press; 1996. p. 101-11. 